\title{
Gentrificación y renovación urbana. Abordajes conceptuales y expresiones en América Latina
}

\author{
Carlos Vergara Constela ${ }^{1}$ \\ cdvc87@gmail.com
}

Recibido: 28 de enero de 2013

Enviado a evaluar: 22 de marzo de 2013

Aceptado: 8 de julio de 2013

\section{RESUMEN}

Enmarcado en la elaboración del trabajo final para la obtención del título de Máster en Estudios Territoriales y de la Población (UAB), el objetivo del presente artículo es exhibir una reflexión teórica sobre las relaciones entre gentrificación y renovación urbana con foco en América Latina. Con la reestructuración productiva a escala global como contexto, se indican los lineamientos primarios entre gentrificación y renovación urbana; luego se abordan los principales enfoques teóricos de la gentrificación residencial; se continúa con una breve exposición de un concepto emergente: gentrificación productiva; en la sección siguiente se hace una sucinta revisión de seis casos de renovación urbana y gentrificación en América Latina generando una posterior discusión teórica en torno a la temática; para finalizar con las principales conclusiones de la reflexión exhibiendo cuatro dimensiones constitutivas del concepto

Palabras clave: Gentrificación, Renovación urbana, Centros históricos, América Latina

\section{Gentrification and urban renewal. Conceptual approaches and expressions in Latin America}

\begin{abstract}
Framed in the final work to obtain the degree of Master of Territorial Studies and Population (UAB), the objective of this article is to show a theoretical reflection on the relationship between gentrification and urban renewal with a focus on Latin America. With the restructuring of production globally and context, the guidelines indicate primary between gentrification and urban renewal; then addresses major theoretical approaches to residential gentrification is followed by a brief summary of an emerging concept: gentrification productive; in section following is a review of six cases of urban renewal and gentrification in Latin America after generating a theoretical discussion about the topic; to end with the main conclusions of the reflection exhibiting four essential dimensions of the concept
\end{abstract}

Key words: Gentrification, Urban Renewal, Historic Downtown, Latin America

\footnotetext{
${ }^{1}$ Sociólogo. Investigador ONG Territorio Sur.
} 


\section{Gentrification et la rénovation urbaine.}

\section{RÉSUMÉ}

Les approches conceptuelles et expressions en Amérique latine

Encadrée dans le travail final pour obtenir le diplôme de Master en Etudes Territoriales et de la population (UAB), l'objectif de cet article est de montrer une réflexion théorique sur la relation entre la gentrification et la rénovation urbaine en mettant l'accent sur l'Amérique latine. Avec la restructuration de la production mondiale et le contexte, les lignes directrices indiquent primaire entre gentrification et la rénovation urbaine; aborde ensuite les principaux approches théoriques de la gentrification résidentielle, est suivie d'un bref résumé d'un concept émergent: la gentrification productive; dans la section Voici un examen de six cas de rénovation urbaine et gentrification en Amérique latine après avoir généré une discussion théorique sur le sujet; pour finir avec les principales conclusions de la réflexion présentant quatre dimensions essentielles du concept

Mots clés: Gentrification, Renouvellement Urbain, Centres Historiques, Amérique latine

\section{CONTEXTO URBANO GLOBAL Y TRANSFORMACIÓN URBANA A TRES VELOCIDADES (A MODO DE INTRODUCCIÓN)}

La transformación de la economía a escala global durante las últimas cuatro décadas bajo el alero de la globalización no sólo ha conllevado efectos como la pérdida del peso del sector secundario en relación al terciario en lo que respecta a las principales fuentes de empleo, y una influencia notable de las tecnologías de la información y comunicación (TIC's) las cuales han transformado ostensiblemente las relaciones sociales no sólo desde planos económicos y culturales, sino que también ha suscitado transformaciones en la organización de las ciudades. En las últimas décadas hemos presenciado un radical proceso de transformación urbana ${ }^{2}$, donde a influencia de las TICS y la búsqueda de inserción y participación en el circuito mundial de acumulación del capital ha cambiado la estructura y la morfología de las urbes (Sassen, 1991; Soja, 2004; De Mattos et al, 2004).

De Mattos (2006), investigador urbano uruguayo, ha señalado que las metrópolis latinoamericanas se encuentran inmersas en una etapa de reorganización debido a una nueva fase de modernización capitalista. La reestructuración productiva altera la geografía económica a escala global impactando no sólo la estructura urbana y social

\footnotetext{
${ }^{2}$ De todas maneras se debe especificar que las ciudades ya se encontraban en franco proceso de transformación. Por ejemplo, la expansión demográfica entre los años 40 y los 60 produjo una expansión de la población de las ciudades generada por las migraciones campo-ciudad puesto que se veía la ciudad como un espacio de movilidad e integración a los nuevos parámetros sociales (Germani, 1988). En este sentido una de las grandes transformaciones tiene que ver con la creciente suburbanización ya sea de los estratos bajos, porque en el marco de la urbanización de los asentamientos humanos las ciudades poseen una mayor estructura de oportunidades que los sectores agrícolas o bien por las estratos altos que "escapan" del centro y se reubican de manera segregada como émbolo de distinción cultural plasmado de manera territorial.
} 
de las ciudades. Se reconocen por lo menos cinco nuevas tendencias constitutivas de esta nueva fase modernizadora: nuevas estructuras productivas y nuevas dinámicas urbanas; desregulación, mercados de trabajo y desigualdad social; negocios inmobiliarios y nuevos criterios urbanísticos; explosión de movilidad en la nueva morfología urbana; y uniformización del paisaje urbano.

"Las políticas de tendencia de liberación, desregulación y flexibilización, junto a la tercerización de la base económica, impulsaron procesos de desestructuraciónreestructuración de los regímenes laborales existentes, lo que incidió en una creciente des-salarización y precarización de la fuerza de trabajo, acentuando las desigualdades sociales bajo nuevas formas de exclusión, segregación, fragmentación y tugurización, afectando la vida social y la calidad de vida en las grandes aglomeraciones urbanas (...) Aparece la periurbanización y la gentrificación como dos modalidades residenciales que influyen en la nueva morfología urbana" (p.52)

En este marco Donzelot (2004) ha mencionado que la reestructuración enfocada en la tercerización de la economía genera nuevas formas de urbanización con distintas velocidades según las condiciones de vida de los grupos sociales que asumen estos procesos: i) zonas tugurizadas, donde existe un manifiesto deterioro de las condiciones de los inmuebles y de las oportunidades sociales de los habitantes. Son territorios marginados que generalmente cargan con tipificaciones negativas dentro de los imaginarios urbanos circundantes (Gravano, 2005); ii) zonas periurbanizadas, donde predominan las construcciones de baja densidad en un contexto campestre pero con conectividad a través del automóvil con la "ciudad central" y la gama de servicios urbanos y; iii) zonas gentrificadas, generalmente localizadas en los barrios centrales de las ciudades, siendo producto de planes de renovación urbana en el marco de ejes asociativos entre agentes urbanos públicos y privados.

Teniendo presente la importancia sustancial de la gentrificación en la estructuración de las actuales dinámicas metropolitanas, el principal objetivo de esta reflexión radica en presentar un panorama sintético de la gentrificación en América Latina. Para dicho objetivo se proponen cinco apartados que aporten una mirada integral a la problemática presentada. En una primera instancia se exhiben las relaciones entre gentrificación y renovación urbana; luego se abordan los principales enfoques teóricos de la gentrificación residencial; se continúa con una breve exposición de un concepto emergente: gentrificación productiva; en cuarto lugar se hace una revisión de seis casos de renovación urbana y gentrificación en América Latina generando una posterior discusión teórica en torno al tema; para finalizar con las principales conclusiones de la reflexión exhibiendo cuatro dimensiones constitutivas del concepto. 


\section{GENTRIFICACIÓN Y RENOVACIÓN URBANA ¿RELACIONES (IN)VISIBLES?}

Hemos señalado que la imposición de nuevos parámetros establecidos por el cambio del modelo global de acumulación centrado en las ciudades genera que éstas busquen nuevas formas para insertarse en los circuitos económicos globales. En este sentido, los planes de renovación urbana de las áreas centrales se conciben como estrategias posindustriales de reconversión de los modelos productivos de las ciudades, teniendo como finalidad la puesta en valor productivo de las condiciones de centralidad provistas por éstas áreas (Luque, 2008).

En una lógica similar Herce (2005) ha mostrado, a través de investigaciones realizadas en el área metropolitana de Barcelona, que la implantación de políticas de corte urbanístico en determinadas áreas de la ciudad genera cambios tanto para el tejido social-urbano de la ciudad, como en los grupos que habitan las áreas de intervención; existe un doble movimiento de migración intra urbana donde se producen desplazamientos forzados y buscados hacia la periferia, según las condiciones socioeconómicas de los grupos sociales, y un retorno al centro de habitantes de estratos medios y medios altos de menor longevidad y con estructuras familiares menos tradicionales.

Por otro lado existen enfoques que ponen énfasis en la renovación urbana de barrios de ciudades que durante la época fordista basaron su desarrollo en los puertos. Al respecto Talesnik y Gutiérrez (2002), a través de la ejemplificación en ciudades como Baltimore, Sidney, Barcelona y Buenos Aires, señalan que rehabilitación de los bordes costeros o frentes de agua también llamados waterfront, han sido operaciones urbanísticas con vastas consecuencias negativas en lo social y urbano. Algunas de ellas tienen relación con la creación grandes centros comerciales en antiguas zonas de uso portuario, alterando las cualidades paisajísticas predominantes (Muñoz, 2008), creando y fortaleciendo espacios de interés turístico en los entornos, alza del precio de suelo y expulsión de habitantes que no tienen la capacidad de hacerse cargo de la nueva realidad socioeconómica de la zona.

La experiencia latinoamericana muestra que los planes de renovación urbana tienen como consecuencias principales la pérdida sustancial de población en la zona intervenida, rehabilitación de inmuebles de uso residencial para uso comercial y cambios socioeconómicos y demográficos en la composición familiar de los nuevos habitantes: elementos que en su integralidad y relación hablan de procesos de gentrificación (Sandroni, 2006; Carrión, 2010, Contreras, 2011).

En relación con lo anterior, Sandroni (2006) ha sostenido que la relación entre renovación urbana y gentrificación -entendida como consecuencia negativa- es premeditada ya que la renovación vendría a constituir se como un estimulo para cambiar el uso de barrios degradados y aumentar la inversión privada en ellos. 


\section{GENTRIFICACIÓN: UN CONCEPTO EN DISPUTA}

Desde el pionero trabajo de Glass (1964) en el centro de Londrés, el abordaje conceptual de la gentrificación no se manifiesta de manera unilateral, sino que podemos encontrar dos grandes tendencias que no solo sustentan los estudios, sino que también expresan perspectivas desde donde entender la realidad social y urbana. Si bien ambas tendencias convergen en la expulsión de habitantes de las áreas centrales, la matriz de la explicación tienen distintos orígenes: por un lado se encuentran quienes explican la gentrificación acentuando la formación de una demanda de vida en el centro nacimiento de una "nueva clase urbana"- y por otro lado, quienes señalan que no es una nueva clase la que emerge, sino que agentes urbanos públicos y privados producen una oferta para habitar en el centro de la ciudad, sustentada por operaciones urbanas y planes de revitalización que cambian la percepción de las áreas centrales y el valor de suelo. En otras palabras, las diferencias de los abordajes conceptuales radican en la identificación de los agentes gentrificadores.

Por la forma en cómo se ha trabajado el artículo, la gentrificación sólo pareciera tener ribetes residenciales. No obstante, geógrafos catalanes han acuñado el concepto de gentrificación productiva, donde se pone acento en los mecanismos y consecuencias de planes de renovación que producen un cambio en los usos de suelo y por tanto un reemplazo irrestricto de ciertas actividades económicas, generando la expulsión de éstas en determinadas zonas (Dot, Casellas y Pallares, 2012).

En el presente apartado primero revisaremos las teorías que ponen acento en la construcción de una demanda por vivir en el centro, luego revisaremos las teorías que resaltan la articulación de una oferta, después se repasarán teorías más eclécticas que van conjugando teorías de oferta y demanda, para finalmente revisar el enfoque de gentrificación productiva.

Teorías enfocadas desde la demanda. Entre los enfoques que proponen como el principal agente gentrificador la construcción de una demanda de población que desea volver de los suburbios a vivir al centro encontramos diversas investigaciones. Ley (1980), quien realizó un estudio en Vancouver, Canadá, durante la década de 1970 sostiene que el cambio de habitantes de la zona central se produce debido a la existencia de agentes con una nueva ideología liberal proveniente del fortalecimiento del sector de servicios en la economía de las ciudades, donde la alta calificación, estatus y nivel profesional demandan espacios en el centro de la ciudad, con el fin de llevar a cabo una "vida urbana" que promueve elementos culturales ligados al hedonismo. Ley (1980) reconoce la emergencia de conflictos urbanos ya que la llegada de nuevos habitantes implica la expulsión de otros. Lo anterior se vería fortalecido por el cambio de predominancia en la fuerza de trabajo, donde proliferan profesionales medios y altos en un contexto de cambio del modelo productivo fordista al posfordista. Hamnett (1991) ha profundizado en los cambios de la composición de la fuerza de trabajo y sus expresiones socio-territoriales, indicando la emergencia de una nueva clase urbana que ante el detenimiento del proceso de suburbanización, demanda viviendas en zonas céntricas de la ciudad. 
En una tónica similar pero con algunos matices diferentes, Hackworth (2002) quien ha realizado sistemáticos estudios urbanos en la ciudad de Nueva York, reconoce la relevancia de los agentes privados y públicos en la producción de gentrificación y la agudización del rent gap, no obstante sus explicaciones ponen énfasis en el fortalecimiento de una clase urbana que demanda habitar las zonas centrales de la ciudad.

Teorías enfocadas desde la oferta. En contraposición con las anteriores teorías que explican la gentrificación, Smith (1979) ha señalado que más que una vuelta de la población al centro de la ciudad, lo que retorna en gran medida es el capital productivo. El geógrafo escocés desestima las teorías provenientes de la demanda, donde la principal causa de la gentrificación vendría a ser un cambio de estilo de vida de nuevos profesionales que desean habitar el centro de la ciudad.

Asumiendo una posición "estructural", la definición de los agentes urbanos que promueven la gentrificación aporta mayor claridad para el análisis del proceso. En este contexto Smith (1979) construye la teoría del rent gap para la explicación de la gentrificación donde el fundamento principal radica en la diferencia de la renta potencial del suelo y la renta capitalizada de suelo. En este sentido "la primera se genera cuando un sitio o barrio, dado el actual uso de la tierra es capaz de producir una cierta cantidad de renta de suelo. Sin embargo, por su localización, esa zona podría generar aun mayor renta si tuviese un uso de suelo diferente. La renta potencial es la cantidad que podría ser capitalizada en virtud de un uso de suelo más intenso" (p.543). Entendiendo que las viviendas constituyen un capital fijo que tiende a (des)valorizarse según las condiciones y apreciaciones del entorno en que se ubique (barrio y localización en la ciudad), la aplicación por parte de alianzas entre agentes urbanos públicos y privados de planes de renovación urbana de las áreas centrales contribuyen a generar esta diferencia potencial del beneficio que podría ser obtenido mediante actividades económicas con una mayor beneficio económico (Sandroni, 2006).

De acuerdo a lo anterior, la gentrificación se sustenta en la degradación de los centros de las ciudades durante las fases más importantes del proceso de suburbanización del poblamiento en las ciudades. Por lo mismo Smith (1979) advierte sobre el proceso de depreciación del capital fijo en las áreas centrales sustentado en la tipificación negativa (estigmatización) de barrios, el abandono en algunos casos y la devaluación del precio de los inmuebles. Desde una posición similar, el geógrafo urbano Tom Slater (2009) critica las teorías culturalistas basadas en la construcción de una demanda de vida en el centro, indicando que es erróneo pensar en una clase media fragmentada como agente urbano de reestructuración; la gentrificación no es la única solución para revitalizar las áreas centrales; la existencia de amplia demostración empírica que demuestra que la voluntad de algunos estratos medios y medio alto de volver al centro es producto de la confluencia de agentes urbanos públicos y privados que propician la situación (Marcuse, 1985); y finalmente la negación de los desplazamientos forzados por parte de los nuevos sectores medios. Por lo mismo, Peck y Tickell (2002) señalaron durante la década pasada que la gentrificación es una forma de producción neoliberal del espacio urbano que se encuentra determinada por la adopción de un modelo político-económico de desarrollo que plasma sus mayores 
intervenciones en la producción de nuevas formas de ciudad: correlación perfecta con la estigmatización, periurbanización y gentrificación, tres procesos que van estructurando nuevas ciudades (Donzelot, 2004)

López (2008), geógrafo chileno quien ha aplicado la teoría del rent gap a los procesos de regeneración urbana acaecidos en la zona pericentral sur de la capital chilena, sintetiza de manera adecuada los conflictos teóricos y también políticos, por qué no, de los estudios urbanos sobre gentrificación:

\begin{abstract}
Basadas en el surgimiento de una demanda refinada y postmoderna como causas para el movimiento de "vuelta a la ciudad". Los "culturalistas" criticarán la teoría del rent gap durante los años noventa por su acentuado estructuralismo en comprender la renta como una superestructura económica que determina la gentrificación, sin reconocer los patrones sociales y culturales de consumo, la capacidad de agencia de los inmigrantes de nivel de ingreso medio-alto y sus estilos de vida posmoderna detrás de estos procesos.
\end{abstract}

Saliendo de la aparente dicotomía entre teorías explicativas desde la oferta y desde la demanda, Zukin (1987 y 1989) -socióloga urbana-, ha realizado sistemáticos estudios situados en el este de Manhattan donde integra ambas perspectivas. Parte de los resultados más relevantes radica en la "doble estructuración" del proceso de gentrificación en el área de estudio, es decir, ésta se produce tanto por la acción de una clase media alta de artistas y bohemios, como por la oferta inmobiliaria de agentes urbanos privados y públicos, articulando un barrio con viviendas remodeladas tipo loft, atractivos de ocio cultural y comercio especializado (boutiques, comida extranjera, bed and breakfast, etc.), expulsión de residentes de baja renta e incluso expulsión de artistas de estratos socioeconómicos más desacomodados, consolidando un proceso de renovación urbana donde existen agentes gentrificadores de diversa índole. Algo similar plantean Bianchini y Parkinson (1993) y Nofre y Martín (2009), en estudios sobre regeneración urbana, gentrificación y cultura realizados en antiguas ciudades industriales de Europa occidental y oriental respectivamente, dando cuenta de la relevancia que posee el ocio nocturno y la capitalización comercial por parte de los agentes urbanos de los estilos de vida "posmodernos", para consolidar estrategias de renovación urbana en centros históricos y/o antiguos barrios emblemáticos.

\title{
4. NUEVOS ENFOQUES: RENOVACIÓN URBANA Y GENTRIFICACIÓN PRODUCTIVA
}

Como se mencionó anteriormente, investigaciones de geógrafos catalanes (Dot, Pallares-Barbera, Casellas, 2012) han dado como resultado un nuevo constructo teórico, el cual ha sido definido como gentrificación productiva. Entendiendo que la gentrificación no solo posee expresiones residenciales, se relaciona la expulsión de actividades económicas con las transformaciones de la economía a escala global y las políticas propias de cada ciudad para comprender los cambios de un barrio en específico. 
A partir de estudios realizados sobre el plan urbanístico@22, el cual define un nuevo uso de suelo para el antiguo distrito industrial del Poblenou ubicado en un sector céntrico del área metropolitana de Barcelona, se analizan las principales consecuencias identificando la expulsión de actividades económicas secundarias, a raíz de la especificación del nuevo uso de suelo, el cual se inserta en el amplio espectro de la actividades terciarias, principalmente en el área de la economía del conocimiento y la información. Lo anterior pretende ser sustentado a través de la mayor productividad que poseen estas actividades en el marco del nuevo espacio global de acumulación en la economía de las ciudades. La simbolización de estos procesos se cristaliza con la construcción de hitos físicos -para el caso de Barcelona, la Torre AGBAR-, pretendiendo denotar una nueva fase de modernización de las ciudades. Dot, Pallares-Barbera, Casallas (2012), reconocen las actividades especificadas en el plan@22 como factores gentrificadores; un alza en el precio de suelo reflejo del plan urbanístico aplicado; y desplazamiento productivo donde se reemplazan las actividades industriales por las actividades terciarias. De acuerdo a lo anterior, los autores definen la gentrificación productiva de la siguiente manera:

A diferencia de la deslocalización industrial, no se presenta como un proceso voluntario de relocalización de la actividad impulsado por el potencial dado de las ventajas de la localización productiva. Como se puede apreciar en las definiciones sobre gentrificación, existe un reconocimiento central del desplazamiento, tanto en los casos residenciales como industriales. El desplazamiento es un proceso desarrollado por promotores de bienes inmobiliarios, planificación de la ciudad y propietarios individuales de suelo, entre otros. Las políticas urbanas son centrales en la desindustrialización y son un prerrequisito para el beneficio de aquellos que disponen de capital para invertir en infraestructura física (p.40).

Las implicancias de la deslocalización forzada de las actividades secundarias generan una reconfiguración del espacio urbano de la ciudad. La grandilocuencia de estos planes bajo la impronta de nuevas estrategias de renovación y desarrollo para la ciudad producen un cambio del tejido urbano, no sólo a escala barrial (donde se aplican los planes), sino que también a escala metropolitana.

Si bien el constructo teórico presentado es propio de un determinado contexto espacial y temporal, el espectro global por donde circulan los nuevos bienes de la economía terciaria va sustentando modelos aplicables y replicables, con diversas consecuencias, en otras regiones del planeta. Cabría preguntarse ¿cómo se ha realizado el proceso de inserción a los nuevos circuitos de la economía global desde las grandes metrópolis latinoamericanas? ¿La tendencia sigue hacia la deslocalización industrial o podemos dar cuenta de casos de gentrificación productiva? 


\section{GENTRIFICACIÓN Y RENOVACIÓN URBANA EN AMÉRICA LATINA}

Martínez (2004) parte de la base que las transformaciones urbanas no tienen la misma dinámica y forma en los distintos países que componen América Latina y menos aún si las comparamos con Estados Unidos, Europa Oriental y Europa Occidental; argumento que parece obvio si es que entendemos que la forma de hacer ciudad, los sistemas políticos y la práctica política urbana no han sido idénticas. A su vez, el arquitecto argentino Tella (2005) advierte que la gentrificación es un proceso tardío iniciado desde la década de 1980 a la fecha, es decir, luego de la reestructuración económica a nivel global. De todos modos, sabemos por Borja y Castells (1998), entre los más destacados, que los procesos globales poseen matices diferentes de acuerdo a la especificidad local, por lo mismo no podríamos asumir que el concepto de gentrificación trabajado hasta el momento posee dinámica y expresión idéntica en Europa, Estados Unidos y Latinoamérica, para explicar este fenómeno de las grandes urbes.

Por lo mismo, en el presente apartado se intenta recoger distintos casos de ciudades que se hayan embarcado en procesos de renovación urbana para dar cuenta de las consecuencias de éstos. No habiendo realizado una búsqueda totalmente exhaustiva, porque no es el objetivo primario de este artículo, los casos presentados en la tabla $\mathrm{n}^{\circ} 1$ intentan ser representativos de la realidad urbana de algunas de las metrópolis latinoamericanas.

Tabla 1. Cuadro comparativo de consecuencias de la renovación urbana en ciudades latinoamericanas.

\begin{tabular}{|c|c|c|c|c|}
\hline PAIS & CIUDAD & $\begin{array}{l}\text { LOCALIZACIÓN DE } \\
\text { LA INTERVENCIÓN }\end{array}$ & $\begin{array}{l}\text { AGENTES URBANOS } \\
\text { EJECUTANTES }\end{array}$ & $\begin{array}{l}\text { CONSECUENCIAS } \\
\text { RECONOCIDAS }\end{array}$ \\
\hline ECUADOR & QUITO & Centro Histórico & $\begin{array}{l}\text { UNESCO y Alianza } \\
\text { Público - Privada }\end{array}$ & $\begin{array}{l}\text { Expulsión de habitantes de } \\
\text { bajos ingresos, aumento del } \\
\text { precio de suelo, } \\
\text { boutiquización }\end{array}$ \\
\hline CHILE & SANTIAGO & Pericentro Sur & Alianza Público - Privada & $\begin{array}{l}\text { Aumento del precio de suelo, } \\
\text { densificación, Expulsión de } \\
\text { habitantes de bajos ingresos, } \\
\text { cambio del paisaje urbano } \\
\text { (vivienda en altura) }\end{array}$ \\
\hline ARGENTINA & BUENOS AIRES & $\begin{array}{l}\text { Centro histórico y barrios } \\
\text { adyacentes con antigua } \\
\text { tradición portuaria }\end{array}$ & Alianza Público - Privada & $\begin{array}{l}\text { Expulsión de habitantes de } \\
\text { bajos ingresos, reconversión } \\
\text { del borde costero, } \\
\text { reconversión al ocio } \\
\text { nocturno, aumento del precio } \\
\text { de suelo, boutiquización }\end{array}$ \\
\hline URUGUAY & MONTEVIDEO & Centro Histórico & Alianza Público - Privada & $\begin{array}{l}\text { Expulsión de habitantes de } \\
\text { bajos ingresos, aumento del } \\
\text { precio de suelo }\end{array}$ \\
\hline CUBA & LA HABANA & Centro Histórico & $\begin{array}{l}\text { UNESCO, Estado cubano } \\
\text { y empresas privadas }\end{array}$ & $\begin{array}{c}\text { Densificación del centro } \\
\text { histórico, leve reconversión a } \\
\text { la boutiquización }\end{array}$ \\
\hline BRASIL & $\begin{array}{l}\text { SALVADOR DE } \\
\text { BAHÍA }\end{array}$ & Centro Histórico & $\begin{array}{l}\text { UNESCO, Gobierno de la } \\
\text { Ciudad y empresas } \\
\text { privadas }\end{array}$ & $\begin{array}{l}\text { Expulsión de habitantes de } \\
\text { bajos ingresos, reconversión } \\
\text { al ocio nocturno, aumento } \\
\text { del precio de suelo, } \\
\text { boutiquización }\end{array}$ \\
\hline
\end{tabular}

Fuente: Elaboración propia, a partir de Carrión (2010), López (2008), Lacarrieu et al (2011), Kanai y Ortega-Alcázar (2009), Schelotto (2007), Rodríguez (2007), Nobre (2002) y Ormindo de Azevedo (2009) 
Como se puede apreciar en la tabla $\mathrm{n}^{\circ} 1$, nos hemos centrado principalmente en la renovación de los centros históricos de algunas ciudades latinoamericanas, salvo en el caso de Santiago de Chile, donde, a pesar que existen experiencias de rehabilitación de la zona central (Contreras, 2011), hemos resaltado los planes de renovación en la zona pericentral sur ${ }^{3}$. Pues bien ¿Por qué el centro histórico como objeto de la renovación? ¿Qué características comunes poseen los centros históricos de América Latina que abogan por renovación urbana? ¿Realmente la necesitan o estamos en presencia de estrategias de otra índole? El urbanista ecuatoriano Fernando Carrión (2010) nos entrega algunas vías de acceso para esta repensar esta problemática:

"La distinción entre centro histórico y centro urbano, que se produce gracias a la pérdida de las funciones de centralidad del área matriz en beneficio del nacimiento de una nueva, conduce a dos posibilidades: a) a una centralidad compartida, donde la histórica mantiene algunas funciones que le dan vida y otras que se desplazan para conformar una nueva; y b) a la pérdida total de las funciones de centralidad que puede conducir a su disolución o, en el mejor de los casos, a su conversión en un barrio histórico que carece de centralidad urbana" (p.190)

De acuerdo a lo señalado en la cita anterior, el desplazamiento de las funciones urbanas a otras zonas promueve el reconocimiento de ciertas condiciones históricas del área fundacional de la ciudad. No es casualidad que diversos investigadores de áreas centrales latinoamericanas (Sandroni, 2006; Carrión, 2010; Contreras, 2011) converjan en que la pérdida de funciones urbanas es uno de los factores que comienza a estructurar el deterioro de estos barrios, donde la tugurización, estigmatización o tipificación negativa de éstos va restándole relevancia funcional dentro del espacio urbano. En este sentido, los planes de renovación urbana vendrían a intentar revalorizar estas áreas.

Enmarcados temporalmente desde la década de 1980 en adelante, es decir, en franca época de reestructuración económica productiva de las metrópolis latinoamericanas, la búsqueda de revitalizar estas zonas no tiene tanta relación con volver a dotarlas de centralidad urbana, sino que la búsqueda va en la vía de integrarse al circuito económico global, a través del turismo. En directa relación con lo anterior, Donaire (1998), geógrafo de la Universidad de Girona, señala que en un contexto productivo posfordista existe una universalización de los espacios turísticos, donde la emergencia de lo patrimonial y su consecuente valorización adquiere inusitada relevancia como estrategia de reconversión de barrios y ciudades con tales condiciones.

De todas maneras cabe seguir señalando algunas especificidades de los planes de renovación y sus consecuencias. Según lo señalado en la tabla $n^{\circ} 1$ sólo el caso de

\footnotetext{
${ }^{3}$ La decisión también posee un sustento metodológico ya que la práctica investigativa nos sugiere exponer un caso que siga la tendencia general pero que posea un especificidad que sirva para algún tipo de comparación (Becker, 2010).
} 
Santiago de Chile estudiado por López (2008) presenta cambios drásticos en el paisaje urbano, donde se puede apreciar una mayor construcción en altura, a diferencia de las otras zonas, las cuales están sujetas a normativas de preservación de los inmuebles que impiden un cambio del paisaje urbano en esta perspectiva. En relación con lo anterior, las zonas con denominación UNESCO más el caso de Buenos Aires ${ }^{4}$, también muestran cambios en el paisaje urbano pero en otro sentido: sin construcción en altura pero con transformación de antiguos inmuebles en hoteles, restaurantes de comida extranjera, comercio de souvenirs, boutiques de ropa vintage, etc., proceso de transformación del paisaje urbano que Carrión (2007) denomina boutiquización. En otras palabras, lo planteado por el urbanista ecuatoriano es una consecuencia de la estrategia de reconversión de estas zonas donde la prioridad no está en el uso residencial sino que en el uso turístico, por lo que se necesita dotar a los barrios de cierto equipamiento específico.

Por lo mismo, no es casualidad que el caso de La Habana no muestre expulsión de residentes y sólo una leve boutiquización. Se entiende que los planes de renovación urbana en determinadas zonas de ciudades se encuentran sujetos a condicionantes más globales como el modelo de desarrollo de cada país.

Hubo centros históricos que, como consecuencias de los tardíos procesos de modernización capitalista que vivieron, la crisis de la centralidad histórica se desató posteriormente (Quito, La Habana) a lo que ocurrió en las ciudades metropolitanas de rápida urbanización (Buenos Aires, Río de Janeiro) (Carrión, 2010; p.191).

A diferencia de Quito, donde durante la década de los noventa estuvo sometido a influencias neoliberales, se produjo expulsión de habitantes, boutiquización y aumento del precio de suelo en la zona patrimonial; La Habana, sostenida en un modelo de país de corte más socialista, no presenta expulsión de residentes, al contrario, muestra densificación del centro histórico y solo leve transformación del uso de suelo urbano.

Pasando a otra dimensión, podemos establecer que los agentes urbanos que promueven y ejecutan las intervenciones en materia de renovación urbana vienen del mundo público y del mundo privado, dejando a la sociedad civil en una posición dominada, tal como ha señalado González (2011) en estudios realizados sobre agentes y renovación urbana. La UNESCO, como agente internacional, también posee un rol fundamental en la legitimación de lo patrimonial y en la potenciación de la "imagen ciudad" o city marketing, la cual publicita los atractivos que merecen ser conocidos dentro de la lógica turística (Yúdice, 2008). Además la denominación UNESCO se convierte en una especie de "aval" para la obtención de créditos de cooperación

\footnotetext{
${ }^{4}$ El caso de Buenos Aires tiene otra particularidad que precisa ser descrita: si bien no existen estudios sobre aquello, la zona llamada Puerto Madero pudo estar sujeta a procesos de gentrificación productiva puesto que, además de la reconversión del borde costero, lo que aminoraría el uso portuario, en determinadas áreas se produjo un cambio de normativa respecto al uso de suelo, donde se privilegian actividades económicas terciarias en detrimento de las portuarias.
} 
internacional (Banco Interamericano de Desarrollo - BID) para el desarrollo de actividades productivas en los espacios patrimoniales ${ }^{5}$.

Más allá que la tabla $n^{\circ} 1$ es un resumen muy acotado de ciertos aspectos de los procesos de renovación urbana en algunos centros históricos de Latinoamérica, la dinámica de las ciudades plantea ciertas problemáticas comunes respecto a la organización y localización de la población en general; existe una nueva estructuración de la población en la ciudad donde se consolidan y expanden los barrios "tugurizados", áreas periurbanas y barrios gentrificados. Si bien ya contábamos con el argumento de Donzelot (2004) quien hablaba de las tres velocidades de transformación de las ciudades, Martínez (2004), urbanista brasilero, ha señalado grosso modo algunas consecuencias de los planes de renovación urbana en ciudades latinoamericanas: expulsión de población de menores recursos; rehabilitación de viejas viviendas degradadas; nuevos emprendimientos comerciales generalmente localizados en viviendas rehabilitadas dentro de barrios en proceso de gentrificación; nueva concepción del espacio público en la ciudad; y reconversión de áreas industriales en nuevos equipamientos urbanos o actividades productivas terciarias.

Por lo tanto abrir preguntas a una discusión más amplia -claramente no zanjada en esta reflexión- sobre los planes de renovación urbana y las actuales transformaciones de las metrópolis latinoamericanas puede ser útil para elaborar nuevos focos investigativos ¿Qué tanta injerencia tienen los planes de renovación urbana de un barrio central en la reorganización de la población y el poblamiento dentro de una gran ciudad? ¿Se producen nuevas migraciones intra o inter urbanas? ¿Cambian las lógicas de movilidad? ¿Aumenta o disminuye la segregación residencial socioeconómica? Son preguntas que pueden guiar investigaciones y/o reflexiones sobre antiguas y nuevas dinámicas sociales y urbanas dentro de algunas de las grandes ciudades de Latinoamérica.

\section{REFLEXIONES FINALES (A MODO DE CONCLUSIÓN)}

Luego de la revisión de algunos de los impacto del nuevo modelo socioeconómico global en la dinámica social y urbana; elementos de la relación ente planes de renovación urbana y gentrificación; y abordajes conceptuales provenientes de los principales investigaciones realizadas en ciudades europeas, norteamericanas y latinoamericanas, podemos reconocer la existencia de cuatro grandes dimensiones que, en su integralidad y capacidad de inter relación, son atingentes para el estudio de la gentrificación, a propósito de los planes de renovación urbana en determinadas zonas de la ciudad. Expulsión de residentes de menores ingresos, alza del precio de suelo,

\footnotetext{
${ }^{5}$ De los casos mencionados en la tabla $n^{\circ} 1$, sólo La Habana no solicitó un crédito de inversión al BID, lo que de alguna manera muestra que el proceso de patrimonialización tiene componentes globales muy semejantes a los casos de Salvador de Bahía en Brasil, o recientemente Valparaíso en Chile, pero aspectos locales como el modelo de desarrollo nacional también posee una injerencia relevante como consecuencias del establecimiento de las estrategias de renovación urbana.
} 
transformación del uso de suelo y transformación del paisaje urbano, van marcando las transformaciones de las ciudades, a raíz de la gentrificación y la renovación urbana.

Expulsión de residentes. A través de la comparación de estadísticas desagregadas a nivel barrial, De Mattos et al (2004) y Contreras (2011) señalan que se puede determinar la densidad población del barrio y la existencia de cambios sustanciales en la composición social de los barrios, identificando qué tipo de habitantes ha dejado el barrio y cuáles han llegado. Así como a través de metodologías cualitativas como entrevistas semi estructuradas y relatos de vida, responder a preguntas de caracterización de los nuevos habitantes ¿de dónde provienen? ¿Cómo nace la motivación de habitar las zonas centrales de la ciudad? ¿Qué elementos poseen estas zonas que generan el deseo de ser habitadas?

Alza del precio de suelo. Como señala Smith (1979) en su teoría sobre el ciclo de la desvalorización y la relación entre renta fija y renta variable, la comparación pre y post aplicación de planes de renovación urbana del precio de suelo de ciertos barrios, o bien localización de zonas con precios de suelo más altos que la media o mediana de la ciudad, pueden ser vías de acceso para la identificación de barrios en proceso de gentrificación residencial o productiva. En el caso de los procesos de renovación urbana, la recuperación de antiguas teorías de la geografía económica sobre localización y valor de suelo (Von Thunen, 1863) puede aportar marcos interpretativos y referenciales interesantes en esta nueva etapa de los centros históricos y centros urbanos de las ciudades latinoamericanas.

Cambio del uso de suelo. El cambio de normativa del uso del suelo propuesto por algunos planes urbanísticos o el cambio porcentual de uso de suelo comercial respecto al residencial en zonas de uso mixto van dando cuenta de transformaciones urbanas que pueden ser analizadas desde una perspectiva de gentrificación residencial o productiva. En este caso se quiere volver a poner énfasis sobre el constructo teórico planteado por Dot, Pallares-Barbera y Casellas (2012), donde se aborda la expulsión de actividades productivas para la llegada de nuevas.

Transformación del paisaje urbano. Los cambios en la matriz paisajística de ciertas zonas vienen a ser el correlato o la cristalización de ciertos cambios a nivel barrial y a nivel de ciudad, que no sólo hablan de un barrio con una nueva dinámica, sino que también hablan de la transformación de la identidad territorial de un lugar (Nogué, 2005).

A fin de cuentas cabe mencionar que el estudio de la gentrificación es una ventana para introducirnos en la nueva estructuración de las ciudades. Conocer los procesos de gentrificación es conocer una parte de la dinámica de la ciudad, pero no es lo único, porque como vimos, en Latinoamérica ésta se produce principalmente en los centros históricos. Entonces, si damos cuenta de expulsión de actividades productivas o de residentes quiere decir que hay relocalización de actividades y/o habitantes. Teniendo en cuenta problemáticas de índoles no directas, pero totalmente relacionadas a los estudios urbanos como la falta de empleo, la precarización de éste, las pautas de movilidad, la concentración de riqueza y pobreza, la sostenibilidad de la ciudad, entre los temas más destacados, estudiar la gentrificación es sólo una parte de las 
nuevas dinámicas que están estructurando el espacio urbano, en constante cambio, de las metrópolis latinas.

\section{BIBLIOGRAFÍA}

BECKER, H. (2010). Trucos del oficio: como conducir su investigación en ciencias sociales. Buenos Aires, Siglo XXI Editores.

BIANCHINI, F., y PARKINSON, M. (1993). Cultural policy and urban regeneration: the west European experience. Manchester, University Press.

BORJA, J., y CASTELLS, M. (1998). Local y global. La gestión de las ciudades en la era de la información. Taurus: Madrid, España.

CARRIÓN, F. (2007). "El financiamiento de la centralidad urbana: el inicio de un debate necesario", en CARRIÓN, F. (editor) (2007). Financiamientos de los centros históricos de América Latina y el Caribe. Quito, FLACSO - Lincoln Institute of Land Policy.

CARRIÓN, F. (2010). El laberinto de las centralidades históricas en América Latina. Quito, Ministerio de Cultura.

CLARK, E. (1988). The rent gap and transformation of the built enviroment: case estudies in malmö. Geografska Annaler. Series B, Human Geography, 70, (2), pp.241-254.

CONTRERAS, Y. (2011). La recuperación urbana y residencial del centro de Santiago: Nuevos habitantes, cambios socioespaciales significativos. Revista EURE (Vol. XXXVII, N¹12), pp. 89-113.

DE MATTOS, C; RIFFO, L; YÁÑEZ, G; SALAS, X. (2004). Reestructuración del mercado metropolitano de trabajo y cambios socio territoriales en el Gran Santiago. Santiago, Instituto de Estudios Urbanos y Territoriales - INE. PUC.

DE MATTOS, C. (2006): "Modernización capitalista y transformación metropolitana en América Latina. Cinco tendencias constitutivas". En: GERAIGES, A., ARROYO, M., y SILVEIRA, M. (2006): America Latina: cidade, campo e turismo. Sao Paulo, CLACSO.

DONAIRE, J (1998). La reconstrucción de los espacios turísticos. La geografía del turismo después del turismo. Revista Sociedade e Territorio, $\mathrm{n}^{\circ} 28$. Universidade Federal do Rio Grande do Norte: Río Grande do Norte, Brasil.

DONZELOT, J. (2004). La ville à trois vitesses: relégation, périubanisation, gentrification. Revista Esprit, Paris, n. 303, marzo-abril.

DOT, E., PALLARES, M., y CASELLAS, A. (2012). Gentrificació productiva, desindustrialització i relocalització industrial. Treballs de la Societat Catalana de Geografia, núm. 73, juny 2012, pp. 27-52

GLASS, R. (1964). London: Aspects of Change. London: Centre for Urban Studies and MacGibbon and Kee

GRAVANO, A. (2005). El barrio en la teoría social. Buenos Airesm Espacio Editorial. 
GERMANI, G. (1988). "La ciudad como mecanismo integrador". En: BASSOLS, DONOSO y MASSOLLO (1988). Antología de Sociología Urbana. México DF, Universidad Nacional Autónoma de México, Colección de Ciencias Sociales.

GONZÁLEZ, F. (2011). "La disputa por la gestión de las ciudades: la reconstrucción social-urbana del campo de luchas en Valparaíso". En BAILEY, G., ESPINOZA, F., CARROZA, N., y VALENZUELA, F. (2011). Valparaíso en Transito: Perspectivas de una nueva sociología de la ciudad. Valparaíso, Editorial Puntángeles, pp.67-82.

HACKWORTH, J. (2002). Postrecession gentrification in New York City. Urban Affairs Review, 37, (6), pp. 815-843.

HAMNETT, C. (1991). The Blind Men and the Elephant: The Explanation of Gentrification. Transactions of the Institute of British Geographers, New Series, $16,(2)$, pp. 173-189.

HERCE, M. (2005). Urbanización, precios del suelo y modelo territorial: la evolución reciente del área metropolitana de Barcelona. Revista EURE (Vol. XXXI, Nº 93), pp. 35-51

KANAI, M., y ORTEGA-ALCÁZAR, I. (2009). The prospects for progressive cultura-led urban regeneration in Latin America: cases from México City and Buenos Aires. International Journal of Urban and Regional Research, Vol. 33.2, pp.483-501.

LACARRIEU, M., GIROLA, M., THOMAZ, A., YACOVINO, M., LEKERMAN, V., y CROVARA, M. (2011). Procesos de recualificación y relegación en la ciudad de Buenos Aires. Repensando la noción de ciudad-fragmento y la despolitización de lo urbano. Revista Argumentos UAM-X, vol. 66, año 24, pp.15-34.

LEY, D. (1980). Liberal ideology and the postindustrial city. Annals of the Association of American Geographers, 70, (2), pp: 238-258.

LÓPEZ, E. (2008). Destrucción creativa y explotación de brecha de renta: discutiendo la renovación urbana del peri-centro sur poniente de Santiago de Chile entre 1990 y 2005. Scripta Nova. Revista Electrónica de Geografía y Ciencias Sociales, vol. XII, núm. 270 (100).

LUQUE, E. (2008). Globalización y ciudad: la reinvención de espacios urbanos en América Latina. Anuario de Estudios Americanos, 65, (2), pp. 265-287.

MARCUSE, P. (1985). Gentrification, abandonment and displacement: connections, causes and policy responses in New York City, en: Journal of Urban and Contemporary Law, 28, pp. 195-240.

MARTÍNEZ, S. (2004). "A gentrification: conceito e método". En: ALESSANDRI, A. \& CABRERA, C. (2004). Urbanizacao e mundializacao, estudios sobre a metrópole. Sao Paulo, LABUR, USP.

MUÑOZ, F. (2008). Urbanalización: paisajes comunes, lugares globales. Gustavo Gili: Barcelona.

NOBRE, E. (2002). Urban regeneration experiencies in Brazil: historical preservation, tourism development and gentrification in Salvador da Bahia. URBAN DESIGN International, 7, pp.109-124. 
NOFRE, J; \& MARTÍN, J. (2009). Ocio nocturno, gentrificación y distinción social en el centro histórico de Sarajevo. Revista Anales de Geografía, 29, (1), pp.91-110.

NOGUÉ, J (2005). Paisatge i identitat territorial en un context de globalització. Treballs de la Societat Catalana de Geografía, 60, pp.173-183.

ORMINDO DE AZEVEDO, P. (2009). El centro histórico de Bahía revisitado. Andamios. Revista de Investigación Social, 16, (12), pp.95-113.

PECK, J. \& TICKELL, A. (2002). Neoliberalizing Space. Antipode. A radical journal of geography, 34, (3), pp. 380-404.

RODRÍGUEZ, P. (2007). "Mecanismos financieros aplicados en la rehabilitación del centro histórico de La Habana", En: CARRIÓN, F. (editor) (2007). Financiamiento de los centros históricos de América Latina y el Caribe. Quito, FLACSO - Lincoln Institute.

SANDRONI, P. (2006). Renovación urbana y gentrificación: evaluación de algunos impactos demográficos, sociales y económicos de la operación urbana Faría Lima en la ciudad de San Pablo. Revista Medio Ambiente y Urbanización, 65, (1), pp.109-110.

SASSEN, S. (1991). La ciudad global: Nueva York, Londres, Tokio. Buenos Aires, Eudeba.

SCHELOTTO, S. (2007). "Breves trazos sobre el financiamiento del centro histórico de Montevideo". En: CARRIÓN, F. (editor) (2007). Financiamiento de los centros históricos de América Latina y el Caribe. Quito, FLACSO - Lincoln Institute.

SLATER, T. (2009). Missing Marcuse: on gentrification and displacement city. CITY, 13, (2), pp. 292-311.

SMITH, N. (1979). Toward a theory of gentrification a back to the city movement by capital, not people. Journal of the American Planning Association., 45, (4), pp.538-548.

SOJA, E. (2004). "Seis discursos sobre la postmetrópolis". En: RAMOS, A. (editor) (2004). Lo urbano en veinte autores contemporáneos. Barcelona, Ediciones UPC.

TALESNIK, D. y GUTIERREZ, A. (2002). Transformaciones de frentes de agua: la forma urbana como producto estándar. Revista EURE (Vol. XXVIII, $N^{\circ}$ 84), pp. 21-31.

TELLA, G. (2005). "Rupturas y continuidades en el sistema de centralidades de Buenos Aires". En: WELCH, M. (editor) (2005). Buenos Aires a la deriva. Transformaciones urbanas recientes. Buenos Aires, Editorial Biblos.

VON THUNEN, J. (1863). Der isolerte staat in Beziehung auf landwirtschaft un national ekonomie. Citado por: DOT, E., PALLARES, M., y CASELLAS, A. (2012). Gentrificació productiva, desindustrialització i relocalització industrial. Treballs de la Societat Catalana de Geografia, núm. 73, juny 2012, pp. 27-52

YÚDICE, G. (2008). Modelos de desarrollo cultural urbano: ¿Gentrificación o urbanismo social? Revista Alteridades, 16, (38), pp.47-61.

ZUKIN, S. (1987). Gentrification: culture and capital in the urban core. Annual Review of Sociology, 13, pp. 129-147.

ZUKIN, S. (1989). Loft living. Culture and capital in urban change. New Brunswick, NJ: Rutgers University Press. 